\title{
Electron Correlation and Hund's Rule
}

\author{
J. W. WARNER, * L. S. BARTELL, AND S. M. BLINDER \\ Department of Chemistry, University of Michigan, Ann Arbor, Michigan'48109, U.S.A.
}

\begin{abstract}
s
It is suggested that simple electronic shielding effects induced by wave function antisymmetrization tend to govern the energy ordering of singlet and triplet terms within a two-electron atomic configuration. This approach gives rise to the following alternating rule: For the term of greatest orbital angular momentum within a configuration, the triplet lies below the singlet. The energy ordering reverses for the term of next highest angular momentum, and continues to alternate with each change of one unit in the orbital angular momentum until the term of lowest angular momentum is reached. In an examination of over 600 energy levels of the elements and their ions, the alternating rule reliably orders singlet-triplet energy levels in some $90 \%$ of the cases.
\end{abstract}

Il est proposé que des effets d'écran électronique induits par l'antisymétrisation de la fonction d'onde ont une tendance de gouverner l'ordre énergétique du singulet et du triplet pour une configuration atomique à deux électrons. Ceci suggère la règle d'alternance suivante: Pour le terme avec le plus grand moment cinétique d'une configuration le triplet est inférieur au singulet. L'ordre énergétique est renversé pour le terme avec le moment cinétique suivant et cette alternance continue au fur et à mesure que le moment cinétique décroît jusqu'au terme avec le moment cinétique le plus bas. Cette règle est vérifiée dans environ $90 \%$ de 600 cas étudiés.

Es wird vorgeschlagen, dass einfache elektronische, von der Antisymmetrisierung der Wellenfunktion induzierte Abschirmungseffekte eine Tendenz haben die Ordnung der Triplett- und Singulettniveaus in einer atomaren Zweielektronenkonfiguration zu beherrschen. Diese Observation führt zu der folgenden Alternierungsregel: Für den Term mit den höchsten Drehimpuls in einer Konfiguration liegt der Triplett unter dem Singulett. Diese Ordnung wird für den Term mit den nächsthöchsten Drehimpuls umgekehrt, und diese Alternierung setzt mit jeder Veränderung einer Drehimpulseinheit fort, bis der Term mit den tiefsten Drehimpuls erreicht wird. Diese Regel wird in $90 \%$ von 600 Fallen verifiziert.

\section{Introduction}

In recent years, a number of papers have appeared [1-8] in which the traditional interpretation of Hund's rule has been brought into question. Hund's first rule assigns the lowest energy to the term of an atomic configuration that has the highest total spin, the traditional explanation being that the Fermi hole between two electrons of the same spin lowers the interelectronic repulsion, and therefore the energy. As pointed out in the references, however, it is usually found in more accurate calculations that, whereas Hund's rule is obeyed, the total interelectronic repulsion is actually greatest in the term of highest multiplicity.

Table I gives the results of variational calculations on the $1 s 2 p$ and $1 s 3 d$ states of helium using scaled hydrogenic wave functions. These results contain the main qualitative features of more accurate calculations. As already noted, the electrons

* Present address: Department of Chemistry, Haverford College, Haverford, PA 19041 
TABLE I. Effective nuclear charges and interelectronic distances for helium states using nuclear charge scaled hydrogenic wave functions.

\begin{tabular}{|c|c|c|c|}
\hline State & $\begin{array}{l}\text { eff } \\
\text { ls }\end{array}$ & $z_{\mathrm{nI}}^{\text {efE }}$ & $\left\langle\mathrm{r}_{12}\right\rangle(\mathrm{a} \cdot \mathrm{u})$. \\
\hline $1 s 2 p^{3} \mathrm{p}$ & 1.9911 & 1.0892 & 4.674 \\
\hline $1 s 2 p^{1} P$ & 2.0030 & 0.9674 & 5.233 \\
\hline $1 s 3 d^{3} \mathrm{D}$ & 1.9999 & 1.0010 & 10.517 \\
\hline $1 \mathrm{~s} 3 \mathrm{~d}^{1} \mathrm{D}$ & 2.0000 & 0.9996 & 10.531 \\
\hline
\end{tabular}

are closer together on the average in the triplet than in the singlet, and the principal source of this inequality can be seen by examining the effective nuclear charges. In the triplet state, the outer orbital is contracted relative to a hypothetical perfect shielding case in which $Z_{n l}=1$, and the inner $1 s$ orbital is expanded relative to $Z_{1 s}=2$. For the singlet state, the results are opposite. The effect is more pronounced in the outer orbital, so that the net effect is that the triplet wave function is more compact than the singlet. A brief review of similar results is given in Ref. 9.

Our point of departure is the following shielding argument. The Fermi hole between two electrons of the same spin places the electrons on the average at wider angles in the triplet state than in the singlet state. This is dynamically a more favorable situation, and allows the outer electron to feel more of the nuclear charge in the triplet state. This idea is not new, having been suggested previously by Bartell ${ }^{*}$ and by Shim and Dahl [9]. The advantage of using a shielding argument in the present context is that shielding can be represented by scaling, and the wave function can be made to satisfy the virial theorem [11]. The traditional interpretation of Hund's rule does not satisfy the virial theorem [1]. For concreteness, we shall confine our considerations to the case of two electrons outside of a spherically symmetrical core. Slater has commented on how the two-electron symmetrization conditions are imbedded in a many-electron wave function [12]. It will be shown that, for such two electron configurations, the above shielding argument will sometimes be reversed, and in other cases will break down altogether.

\section{Angular Correlation for Point Hole States}

The angular correlation between two atomic electrons depends to a certain extent on the quantity employed to measure it. We choose to compare the expectation value of $\left(\sin \frac{1}{2} \theta\right)^{-1}$, where $\theta$ is the angle between the electrons at the nucleus. This function is monotonic and one to one on the interval $0 \leq \theta \leq \pi$, and is easily calculated. More importantly, the numbers involved with this measure can

\footnotetext{
* See footnote 6 in Ref. 10.
} 
be applied directly to the construction of a density matrix in Sec. 3. The wave function is taken to be

$$
\psi=\left(1 \pm p_{12}\right) \phi
$$

(here and throughout, upper sign refers to singlet, and lower sign to triplet), where $P_{12}$ is the two particle permutation operator, and $\phi$ is an eigenfunction of $l_{1}^{2}, l_{2}^{2}$, and $L^{2}$ :

$$
\phi\left(\mathbf{r}_{1}, \mathbf{r}_{2}\right)=R\left(r_{1}, r_{2}\right) \sum_{m_{1} m_{2}} C\left(l_{1}, l_{2}, L ; m_{1}, m_{2}, M\right) Y_{l_{1}, m_{1}}\left(\theta_{1}, \phi_{1}\right)-Y_{l_{2}, m_{2}}\left(\theta_{2}, \phi_{2}\right)
$$

Here, we assume $l_{1} \neq l_{2}$, and will call such states point hole states, in reference to the geometry of the Fermi hole. The reason for this distinction will become clear in Secs. 4 and 5 , where it will be shown that states with $l_{1}=l_{2}$ must be treated separately because of a different geometry for the Fermi hole. The phase conventions and rules for the Clebsch-Gordan coefficients in (2.2) are as given in Ref. 13. The radial portion $R\left(r_{1}, r_{2}\right)$ is presently assumed to have the requisite degree of detail to represent approximately two electrons outside a spherically symmetrical electron core. Then it is shown in the Appendix that

$$
\left\langle\left(\sin \frac{1}{2} \theta\right)^{-1}\right\rangle=A \pm u^{2} B
$$

where $A$ and $B$ depend only upon angular integrations, and $u^{2}$ is the square of the radial overlap

$$
u^{2}=2 \int_{0}^{\infty} r_{1}^{2} d r_{1} \int_{0}^{\infty} r_{2}^{2} d r_{2} R\left(r_{1}, r_{2}\right) P_{12} R\left(r_{1}, r_{2}\right)
$$

In accordance with the results of Table $\mathrm{I}, u^{2}$ is a different function for singlet and triplet. It is also shown in the Appendix that $B$ is positive for the lowest and highest orbital angular momentum terms of a configuration. Moreover, in all cases studied so far-those included in Table II, which comprise the overwhelming majority of relevant cases for which experimental data are easily available $-B$ follows an alternating rule, being positive for the term of highest orbital angular momentum within a configuration, negative for the next highest term, etc., alternating in sign with each change of one unit in the total orbital angular momentum. Equation (2.3) and the alternating rule for $B$ are the relations sought in order to apply the shielding argument of Sec. 1. So assuming the angular effect determines the energy ordering, the triplet state is expected to lie below the singlet for the term of highest angular momentum, and the ordering is expected to reverse as the orbital angular momentum changes by one unit.* However, more insight may be gained using a simple analytic form for $R\left(r_{1}, r_{2}\right)$ capable of representing a

\footnotetext{
* The alternating rule has been noted empirically for the spectrum of scandium in Ref. $15 \mathrm{a}$; it is also mentioned in connection with multiplet theory in Ref. $15 \mathrm{~b}$.
} 
TABLE II. Comparison of alternating rule with experiment for point hole states. An asterisk indicates application of Eq. (2.9). A question mark indicates an experimental uncertainty in the assignment of one of the energy levels. All data from Ref. 14.

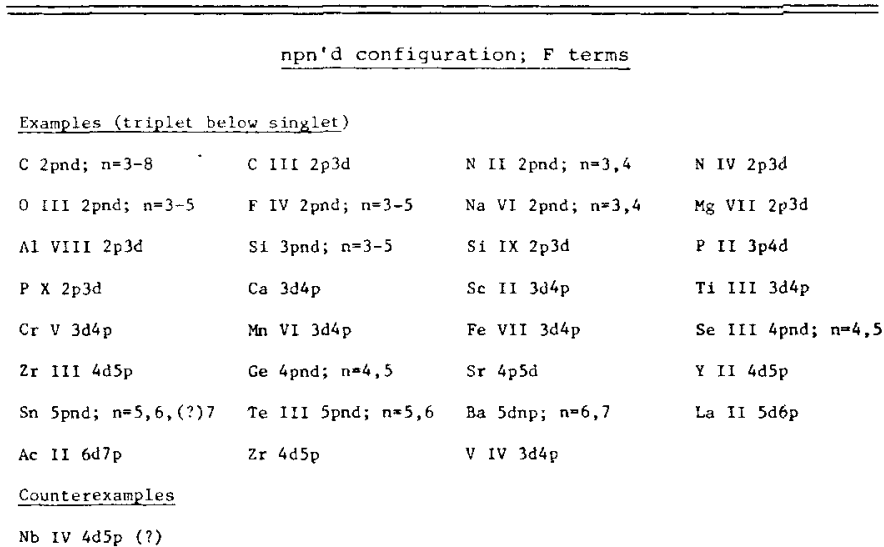

\section{npn'd configuration; D terms}

Examples (singlet below triplet)

\begin{tabular}{|c|c|c|c|}
\hline C 2pnd; $n=3-6$ & C III $2 p 3 d$ & N II $2 \mathrm{pnd} ; n=3,4$ & $N$ IV $2 p 3 d$ \\
\hline O III 2pnd; $n=3-6$ & O V 2 pnd; $n=3-5$ & F iv 2pnd; $n=3-5$ & F VI 2 pnd; $n=3,4$ \\
\hline $\mathrm{Ne}$ V 2 pnd; $\mathrm{n}=3,4$ & Na VI 2 pnd; $n=3,4$ & Na VIII 2pnd; $n=3,5$ & $M g$ VII $2 p 3 d$ \\
\hline Al VIII $2 \mathrm{p} 3 \mathrm{~d}$ & Al $\times 2 p 3 d$ & Si IX 2p3d & S1 XI $2 \mathrm{p} 3 \mathrm{~d}$ \\
\hline Mg IX 2pnd; $n=3-5$ & $P$ IV $3 p 3 d$ & $\mathrm{P} \times 2 \mathrm{p} 3 \mathrm{~d}$ & $P X I I 2 p 3 d$ \\
\hline $\mathrm{Ca} 3 \mathrm{~d} 4 \mathrm{p}$ & Sc II $3 d_{14 p}$ & Ti III $3 d 4 \mathrm{p}$ & V IV $3 d 4 p$ \\
\hline $\mathrm{Cr} v 3 \mathrm{~d} 4 \mathrm{p}$ & Mn VI $3 d 4 p$ & Se III 4pnd; $n=4, * 5$ & $2 \mathrm{r}$ III $405 p$ \\
\hline Nb IV $4 \mathrm{~d} 5 \mathrm{p}$ & Ce $4 \mathrm{p} 4 \mathrm{~d}$ & Sx $4 \mathrm{p} 5 \mathrm{~d}$ & $Y$ II $4 d S_{p}$ \\
\hline Ba $5 d 6 p$ & La II $5 d 6 p$ & & \\
\hline \multicolumn{4}{|l|}{ Counterexamples } \\
\hline Si 3pnd; $n=3-5$ & P II 3pnd; $n=3,4$ & Ge $4 \mathrm{p} 5 \mathrm{~d}$ & As II $4 \mathrm{p} 4 \mathrm{~d}$ \\
\hline Sn Spnd; $n=5,6$ & Te III 5pnd; $n=5,6$ & $\star B a 5 d 7 p$ & Ac II $6 \mathrm{~d} 7 \mathrm{p}$ \\
\hline
\end{tabular}

\section{npn'd configuration; $P$ terms}

Examples (triplet below singlet)

$\begin{array}{llll}\text { C III 2p3d } & \text { N II 2pnd; } n=3,4 & \text { N IV 2p3d } & \text { O III 2pnd; } n=3,4 \\ \text { O V 2pnd; } n=3,4 & \text { F IV 2pnd; } n=3-5 & \text { F VI 2pnd; } n=3,4 & \text { Ne V 2p3d } \\ \text { Na VI 2p3d } & \text { Mg VII 2pnd; } n=3,4 & \text { AI VIII 2p3d } & \text { SI 3pnd; } n=3-5 \\ \text { Si IX 2p3d } & \text { P II 3pnd; } n=3,4 & \text { P IV 3p3d } & \text { Sc II 3d4p } \\ \text { Ti III 3d4p } & \text { V IV 3d4p } & \text { Cr V 3d4p } & \text { Mn VI 3d4p } \\ \text { ZI III 4d5p } & \text { Ce 4pnd; } n=4,5 & \text { Sn 5pnd; } n=5-7 & \text { Te III 5pnd; } n=5,6 \\ \text { Ba Sdnp; } n=6,7 & \text { La II Sd6p } & \text { AC II 6d7p } & \text { 2r 4d5p (?) } \\ \text { P X 2p3d } & \text { Na VIII 2pnd; } n=3,4 & & \end{array}$


TABLE II. (Continued from previous page.)

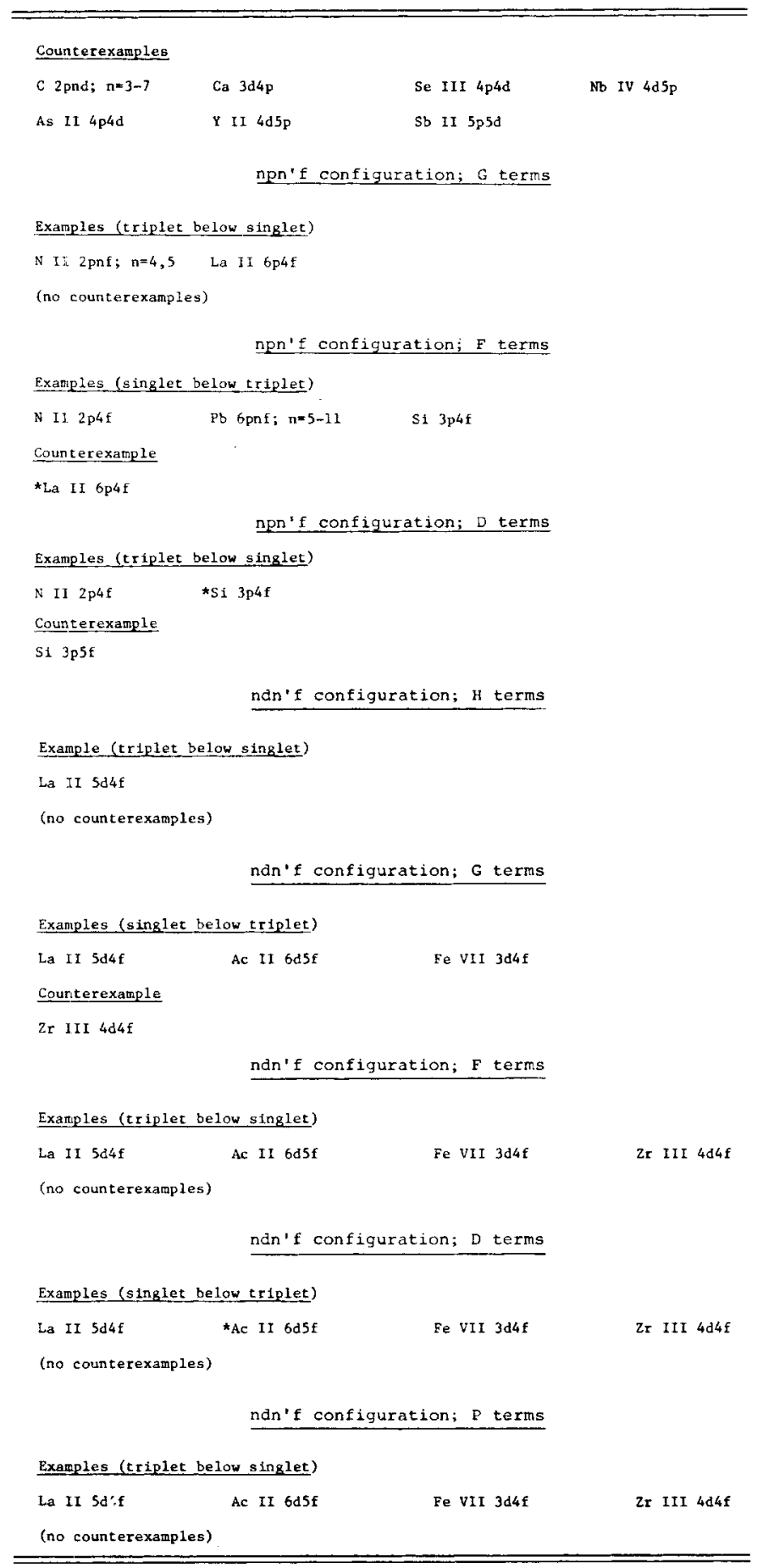


wave function in a central field. Accordingly we take $R$ to be a product of sTos:

$$
R\left(r_{1}, r_{2}\right)=\left(N_{a} r_{1}^{n_{a}-1} e^{-\alpha_{a} r_{1} / n_{a}}\right)\left(N_{b} r_{2}^{n_{b}-1} e^{-\alpha_{b} r_{2} / n_{b}}\right),
$$

where $\alpha_{a}$ and $\alpha_{b}$ are scaling parameters. Letting

$$
S \equiv \frac{2 n_{b}+1}{n_{a} \alpha_{b} / n_{b} \alpha_{a}+1}-\frac{2 n_{\alpha}+1}{n_{b} \alpha_{a} / n_{a} \alpha_{b}+1},
$$

and with the help of (2.4), it is easily shown that

$$
\begin{aligned}
& \frac{\partial u^{2}}{\partial \alpha_{a}}=-\frac{u^{2}}{\alpha_{a}} S, \\
& \frac{\partial u^{2}}{\partial \alpha_{b}}=\frac{u^{2}}{\alpha_{b}} S .
\end{aligned}
$$

Assume first that $B$ is positive; negative values for $B$ will simply reverse our conclusions with respect to singlet and triplet states. In order to have a welldefined inner and outer orbital, let $n_{a}<n_{b}$ and $\alpha_{a}>\alpha_{b}$. Then $S$ is surely positive, and Eq. (2.7) indicates that we may increase the overlap, and therefore the angle between the electrons, in the triplet by increasing $\alpha_{b}$ and decreasing $\alpha_{a}$. In the singlet state, where it is necessary to decrease the overlap in order to increase the angle between the electrons $(B>0)$, it would be necessary to decrease $\alpha_{b}$ and increase $\alpha_{a}$. Furthermore, since $\alpha_{a}>\alpha_{b}$,

$$
\left|\frac{\partial u^{2}}{\partial \alpha_{a}}\right|<\left|\frac{\partial u^{2}}{\partial \alpha_{b}}\right|,
$$

so that rescaling the outer orbital will be more effective in mitigating the angular repulsion than an equivalent rescaling of the inner orbital. These results are qualitatively the same in all respects as those in Table I, and indicate a net contraction of the triplet wave function relative to the singlet $(B>0)$. In order to decide upon the energy ordering, we first note that wave functions of the type (2.5) can satisfy the virial theorem [11]. The contracted triplet, with a higher effective nuclear charge for the outer electron, where the scaling is most pronounced, will have a higher total kinetic energy (which increases as the square of the effective nuclear charge for each of the one-electron orbitals) and therefore the lower energy $(B>0)$.

Table II compares the alternating rule with experiment. The $n s n^{\prime} l$ configurations have been excluded for brevity, because they follow the ordinary Hund rule. All relevant data from Moore's tables [14] have been included. In cases where the singlet is imbedded in the triplet, the triplet terms have been combined, under the assumption that the splitting is entirely spin orbit and is of the form

$$
E_{\mathrm{so}}=\operatorname{const}[J(J+1)-L(L+1)-S(S+1)],
$$

in order to produce one triplet energy independent of spin-orbit coupling. Since (2.9) is an approximation, some of the assignments are possibly open to question. 


\section{Density Matrix Techniques}

A simple visual illustration of the angular correlation can be obtained by constructing an angular density matrix. For this purpose we choose

$$
\left\langle\delta\left(\cos \theta-\cos \theta^{\prime}\right)\right\rangle .
$$

Physically, this quantity represents the probability density as a function of the angle between the two electrons. Making use of (2.2) for the wave function and expanding the delta function in Legendre polynomials

$$
\begin{aligned}
\delta\left(\cos \theta-\cos \theta^{\prime}\right) & =\sum_{l} P_{l}\left(\cos \theta^{\prime}\right) P_{l}(\cos \theta) \\
& =\sum_{l} P_{l}\left(\cos \theta^{\prime}\right) \sum_{m} \frac{4 \pi}{2 l+1} Y_{l, m}^{*}\left(\theta_{2}, \phi_{2}\right) Y_{l, m}\left(\theta_{1}, \phi_{1}\right),
\end{aligned}
$$

we encounter the same angular integrals as in Sec. 2. Study of Eq. (7.1) shows that the $l$ th coefficient in (7.7) must be twice the coefficient of $P_{1}\left(\cos \theta^{\prime}\right)$ in the angular portion of the exchange term of the density matrix. Representing this coefficient by $K_{l}$, and subtracting the triplet density matrix from the singlet in order to magnify the difference gives

$$
\left\langle\delta\left(\cos \theta^{\prime}-\cos \theta\right)\right\rangle_{s}-\left\langle\delta\left(\cos \theta^{\prime}-\cos \theta\right)\right\rangle_{t}=\left(u_{s}^{2}+u_{t}^{2}\right) \sum_{l} K_{l} P_{l}\left(\cos \theta^{\prime}\right),
$$

where the subscripts $s$ and $t$ denote singlet and triplet, respectively. Since this measure depends on the radial overlaps, which will be different for different configurations, we define the quantity

$$
\Delta_{L}\left(\cos \theta^{\prime}\right)=\frac{\left\langle\delta\left(\cos \theta^{\prime}-\cos \theta\right)\right\rangle_{s}-\left\langle\delta\left(\cos \theta^{\prime}-\cos \theta\right)\right\rangle_{t}}{\left(u_{s}^{2}+u_{t}^{2}\right)},
$$

which is scaled to account for these differences. In Figure $1, \Delta_{L}$ is plotted for the $n p n^{\prime} d, n p n^{\prime} f$, and some of the $n s n^{\prime} l$ configurations.

This function has a simple physical interpretation. Anywhere $\Delta_{L}$ has a positive value, the electrons are more likely to be at that angle in the singlet state than in the triplet. Negative values of $\Delta_{L}$ imply a preponderance of the charge density at the corresponding angle in the triplet state. It must be recalled that, while the sum of radial overlaps in (3.4) should be comparable within a configuration, they may change radically between configurations, so the graphs for the $n s n^{\prime} l$ states have each been modulated by a different scale factor in the definition of $\Delta_{L}$.

Most of the graphs clearly indicate a tendency for the electrons to accumulate at close angles in the singlet for the cases where $B>0$, and in the triplet when $B<0$. However, as the degree of oscillation increases, such an interpretation becomes more difficult. In such cases, it is simpler to use Eq. (2.3). Since $P_{l}(1)=1$, the sign of $B$ is the same as that of $\Delta_{L}$ at the right-hand boundary; the close angle behavior of the graphs of $\Delta_{L}$ can thereby be deduced from the alternating rule for $B$. 
(a)

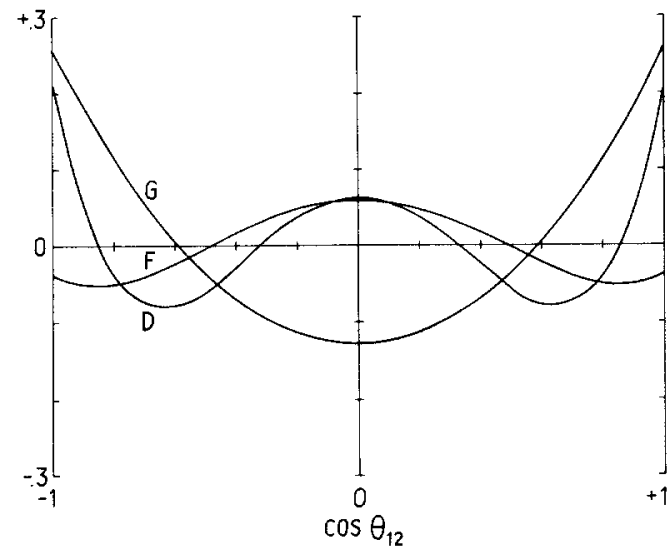

(b)

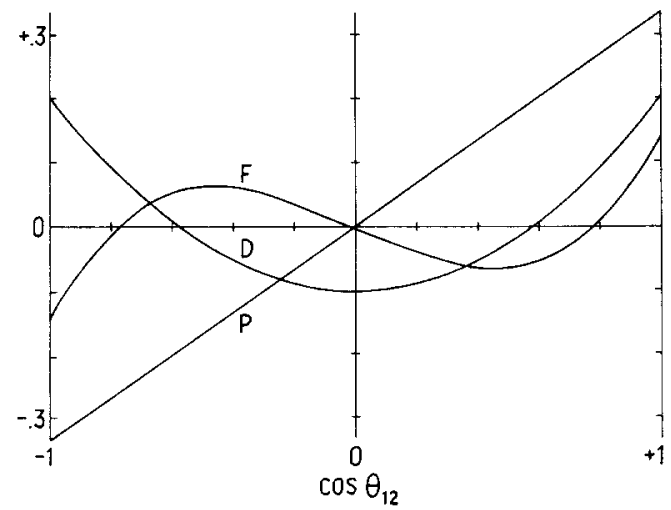

(c)

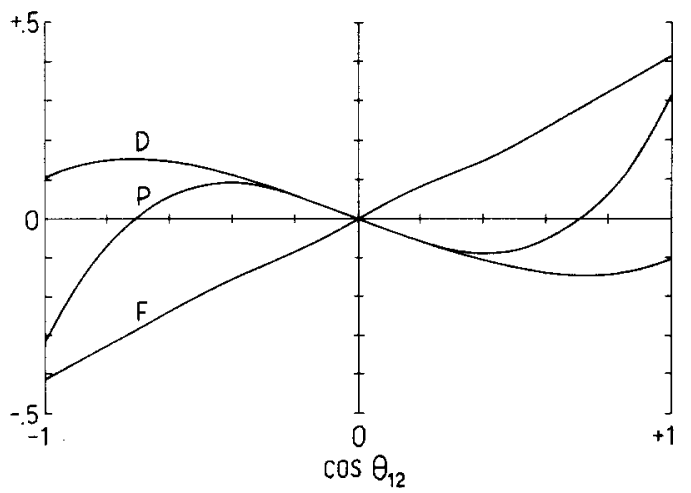

Figure 1. Overlap scaled angular density matrix difference (singlet minus triplet) for some two electron configurations (a) $n p n^{\prime} f$ state; (b) $n s n^{\prime} l$ state; (c) $n p n^{\prime} d$ state. 


\section{Discussion of Surface Hole States}

It remains from Sec. 2 to treat those states made up of two electrons with equal orbital angular momenta. The simplest example of such a state is the $1 s 2 s$ state of helium. A suitable wave function might be constructed in the form

$$
\psi\left(\mathbf{r}_{1}, \mathbf{r}_{2}\right)=N\left[1 s\left(r_{1}\right) 2 s\left(r_{2}\right) \pm 1 s\left(r_{2}\right) 2 s\left(r_{1}\right)\right] \sum a^{e, f, g} r_{1}^{e} r_{2}^{f} r_{12}^{g}
$$

Setting $r_{1}=r_{2}$ then causes the triplet wave function to vanish. The Fermi hole, usually regarded as a small region about the point position of one of the electrons in this case has a much different goemetry; it consists of a spherical surface about the nucleus defined by the radial coordinate of one of the electrons. We shall refer to such states as surface hole states. Because of the spherical geometry of the Fermi hole, these states have no exchange-symmetry induced angular correlation in zeroth order. Rather, they exhibit radial correlation. Because radial wave functions exhibit much greater variety than the angular functions used in Sec. 2, a completely satisfactory treatment of surface hole states has not been accomplished. A shielding argument may in fact be a poor way of describing the difference between the two states. For instance, if both electrons are in $s$ orbitals, the single-configuration wave function of the type (2.2) reduces to

$$
\psi\left(r_{1}, r_{2}\right)=N\left[R\left(r_{1}, r_{2}\right) \pm R\left(r_{2}, r_{1}\right)\right]
$$

Then it is easily shown that

$$
\left\langle 1 / r_{12}\right\rangle=\left\langle 1 / r_{>}\right\rangle
$$

where $r_{>}$is the greater of $\left(r_{1}, r_{2}\right)$. The total atomic potential energy of the two electrons may then be written

$$
\left\langle-\frac{z}{r_{1}}-\frac{z}{r_{2}}+\frac{1}{r_{12}}\right\rangle=\left\langle-\frac{z}{r_{<}}-\frac{(z-1)}{r_{>}}\right\rangle
$$

where $r_{<}$is the smaller of $\left(r_{1}, r_{2}\right)$. Equation (4.4) indicates that the potential energy is equivalent to a system where the outer electron is perfectly shielded by the inner electron. Since this is true for both singlet and triplet states, it does not appear that shielding describes the difference between the two states. Consequently, we defer treatment of surface hole states to a future study, and assume presently that the state that contains the surface hole has the lower energy.

\section{Alternating Rule for Surface Hole States}

We now consider a general configuration in which $l_{1}=l_{2}$. Since we are comparing a singlet state to the corresponding triplet of the same total orbital angular momentum, the case of equivalent electrons is excluded. The wave 
function is constructed according to (2.1) and (2.2) with $l_{1}=l_{2} \equiv l$ :

$$
\begin{aligned}
\phi\left(\mathbf{r}_{1}, \mathbf{r}_{2}\right) & =R\left(r_{1}, r_{2}\right) \sum_{m_{1} m_{2}} C\left(l, l, L ; m_{1}, m_{2}, M\right) Y_{l, m_{1}}\left(\theta_{1}, \phi_{1}\right) Y_{l, m_{2}}\left(\theta_{2}, \phi_{2}\right), \\
P_{12} \phi & =R\left(r_{2}, r_{1}\right) \sum_{m_{1} m_{2}} C\left(l, l, L ; m_{1}, m_{2}, M\right) Y_{l, m_{1}}\left(\theta_{2}, \phi_{2}\right) Y_{l, m_{2}}\left(\theta_{1}, \phi_{1}\right) .
\end{aligned}
$$

Using a symmetry relation of the Clebsch-Gordan coefficients [13] in (5.2) gives

$$
\begin{aligned}
P_{12} \phi & =R\left(r_{2}, r_{1}\right)(-)^{2 l-L} \sum_{m_{1} m_{2}} C\left(l, l, L ; m_{2}, m_{1}, M\right) Y_{l, m_{1}}\left(\theta_{2}, \phi_{2}\right) Y_{l, m_{2}}\left(\theta_{1}, \phi_{1}\right) \\
& =(-)^{L} R\left(r_{2}, r_{1}\right) \sum_{m_{1} m_{2}} C\left(l, l, L ; m_{1}, m_{2}, M\right) Y_{l, m_{1}}\left(\theta_{1}, \phi_{1}\right) Y_{l, m_{2}}\left(\theta_{2}, \phi_{2}\right),
\end{aligned}
$$

so that

$$
\begin{aligned}
\psi\left(\mathbf{r}_{1}, \mathbf{r}_{2}\right)= & N\left[R\left(r_{1}, r_{2}\right) \pm(-)^{L} R\left(r_{2}, r_{1}\right)\right] \\
& \times \sum_{m_{1} m_{2}} C\left(l, l, L ; m_{1} m_{2}, M\right) Y_{l, m_{1}}\left(\theta_{1}, \phi_{1}\right) Y_{l_{1} m_{2}}\left(\theta_{2}, \phi_{2}\right) .
\end{aligned}
$$

The normalization constant is given by

$$
N_{s, t}^{2}=\left(\int\left[R\left(r_{1}, r_{2}\right) \pm(-)^{L} R\left(r_{2}, r_{1}\right]^{2}\right)^{-1}\right.
$$

if $g(\theta)$ is any function of the angle between the electrons, then the difference between the expectation value of $g(\theta)$ for the singlet and the triplet is given by

$$
\begin{aligned}
\langle g(\theta)\rangle_{s}-\langle g(\theta)\rangle_{t}= & N_{s}^{2} \int\left[R\left(r_{1}, r_{2}\right)+(-)^{L} R\left(r_{2}, r_{1}\right)\right]^{2}\left[A^{*} g(\theta) A\right] \\
& -N_{t}^{2} \int\left[R\left(r_{1}, r_{2}\right)-(-)^{L} R\left(r_{2}, r_{1}\right)\right]^{2}\left[A^{*} g(\theta) A\right] \\
& =0
\end{aligned}
$$

where

$$
A=\sum_{m_{1} m_{2}} C\left(l, l, L ; m_{1}, m_{2}, M\right) Y_{l, m_{1}}\left(\theta_{1}, \phi_{1}\right) Y_{l, m_{2}}\left(\theta_{2}, \phi_{2}\right) .
$$

Equation (5.6) shows that there is no difference in angular correlation between singlet and triplet states with $l_{1}=l_{2}$ as a result of symmetrization, within the context of the single configuration approximation. Equation (5.4) shows that the effect of symmetrization is contained in the radial portion of the wave function. Consequently, it is not necessary to consider the angular portion of the wave function in the present context. Squaring (5.4) and integrating over angles then gives

$$
\left|\psi\left(r_{1}, r_{2}\right)\right|^{2}=N^{2}\left[R\left(r_{1}, r_{2}\right) \pm(-)^{L} R\left(r_{2}, r_{1}\right)\right]^{2}
$$


We have regained an equation of the type discussed in Sec. 4 with the addition that the surface hole alternates between the singlet and triplet each time the orbital angular momentum is changed by one unit. The state of greatest orbital angular momentum has the surface hole in the triplet. Thus, with the assumption at the end of Sec. 4, we obtain for the surface hole states the same alternating rule discussed for the point hole states in Sec. 2. Table III compares the rule with experiment. Conditions are the same as for Table II.

\section{Conclusion}

In spite of the simplicity of the present treatment, the alternating rule reliably orders singlet and triplet states of a given orbital angular momentum some $90 \%$ of the time. However, since Hund's rule does not work in all cases, a highly detailed theory of the energy orderings is probably not warranted. We have made no mention of how terms of different orbital angular momentum within a configuration are ordered with respect to each other, but insofar as Hund's second rule

TABLE III. Comparison of alternating rule with experiment for surface hole states. Conditions are the same as for Table II.

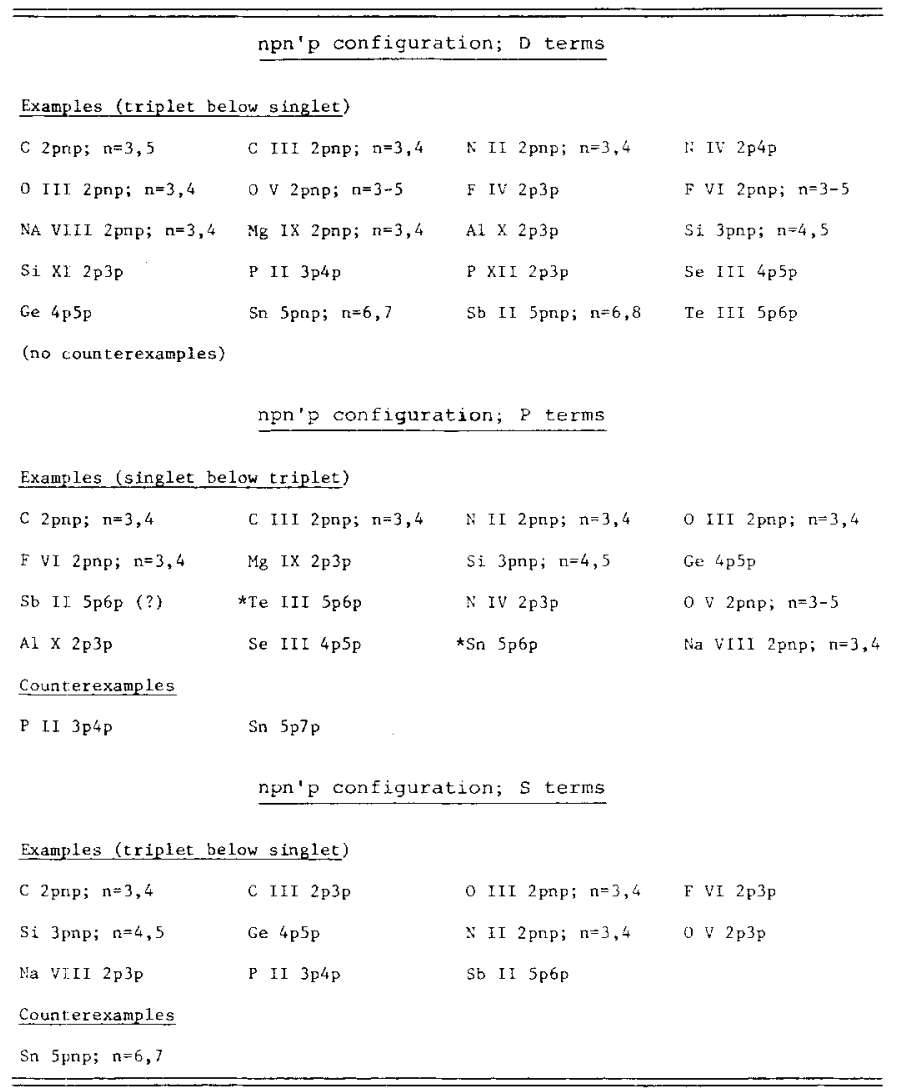


TABLE III. (Continued from previous page.)

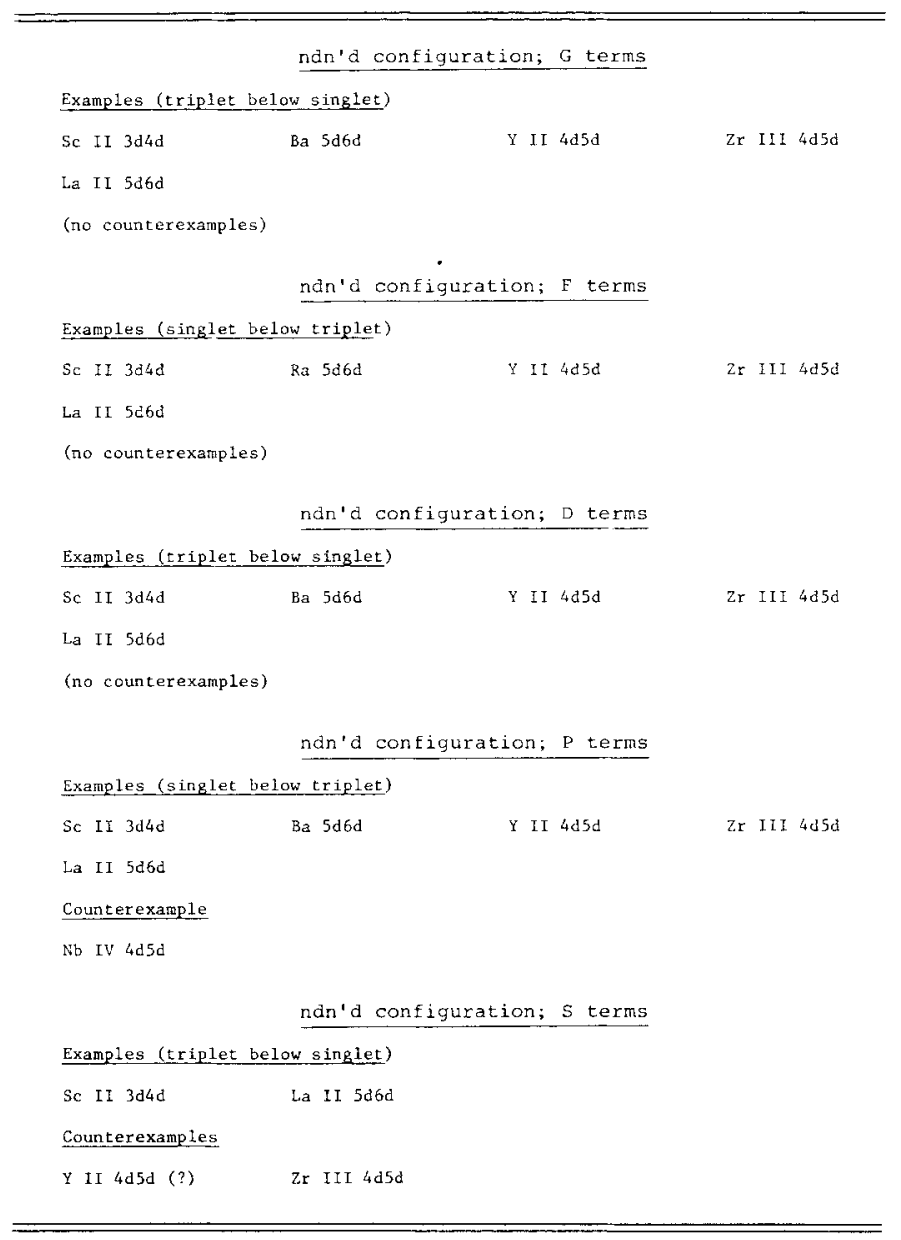

holds, and insofar as the present approach predicts the term of highest orbital angular momentum to have the triplet below the singlet, the ordinary statement of Hund's rule is retained.

The ordinary statement of Hund's first two rules can give the impression that they are independent of each other, a failure of Hund's second rule, for instance, having no effect on the success of Hund's first rule. One aspect of the alternating rule is that these two rules can no longer be considered independent. A failure of Hund's second rule can give rise to a failure of Hund's first rule. Table IV compares the ordering of the singlet $D$ term with the triplet $F$ term for the $n p n^{\prime} d$ configuration; Hund's rules, which assign the lower energy to the triplet $F$ term, work only about $50 \%$ of the time. The singlet $D$ term is as likely to be found lowest as the triplet $F$. 
TABLE IV. Comparison of Hund's second rule with experiment for the $n p n^{\prime} d$ configuration. An asterisk indicates a deviation from the alternating rule.

\begin{tabular}{|c|c|c|c|}
\hline Cases where & $1_{\text {D }}$ lies below ${ }^{3} F$ & & \\
\hline C 2 pad; $n=3-6$ & *Si 3pnd; $n=3-5$ & Se II $3 d 4 p$ & Ti III $3 d^{4} 4 \mathrm{p}$ \\
\hline $\mathrm{CrV} 3 \mathrm{~d} 4 \mathrm{p}$ & $\mathrm{Mg}$ VI $3 \mathrm{~d} 4 \mathrm{p}$ & Fe VII $3 \mathrm{~d} 4 \mathrm{p}$ & $2 \mathrm{r}$ III $4 \mathrm{~d} 5 \mathrm{p}$ \\
\hline Ge $4 \mathrm{p} 4 \mathrm{~d}, * 4 \mathrm{p} 5 \mathrm{~d}$ & $\star \mathrm{Nb}$ IV $4 \mathrm{~d} 5 \mathrm{p}$ & $Y$ II $4 d 5 p$ & La II $5 \mathrm{~d} 6 \mathrm{p}$ \\
\hline$C$ III $2 \mathrm{P}^{3 \mathrm{~d}}$ & $* 2 r<d 5 p$ & $\therefore$ IV $2 \mathrm{p} 3 \mathrm{~d}$ & \\
\hline \multicolumn{4}{|c|}{ Cases where ${ }^{3} \mathrm{~F}$ Iies below ${ }^{1} \mathrm{D}$} \\
\hline$N$ II $2 p 3 d$ & $\mathrm{Ca} 3 \mathrm{~d} 4 \mathrm{P}$ & Se III $4 p^{4} d, 4 p 5 d$ & $\operatorname{sr} 4 d 5 p$ \\
\hline Ba $5 d 7 p, \star 5 d 7 p$ & $\star S n$ sp $5 d, 5 p 5 d$ & $*$ Te III $5 p 5 d, 5 p 6 d$ & $*_{A C}$ II $6 d 7 \mathrm{P}$ \\
\hline $\mathrm{Mg}$ VII $2 \mathrm{p} 3 \mathrm{~d}$ & $\mathrm{Na}$ VI $2 \mathrm{p} 3 \mathrm{~d}$ & F IV 2 pnd; $n=3-5$ & O III $2 \mathrm{pnd} ; \mathrm{n}=3-5$ \\
\hline
\end{tabular}

\section{Appendix: Expectation Value of $\left(\sin \frac{1}{2} \theta\right)^{-1}$}

Since $\frac{1}{2}\left(\sin \frac{1}{2} \theta\right)^{-1}$ is equal to $r_{12}^{-1}$ evaluated at $r_{1}=r_{2}=1$, the expansion in spherical harmonics is

$$
\left(\sin \frac{1}{2} \theta\right)^{-1}=2 \sum_{l} \sum_{m} \frac{4 \pi}{2 l+1} Y_{l, m}^{*}\left(\theta_{1}, \phi_{1}\right) Y_{l, m}\left(\theta_{2}, \phi_{2}\right) .
$$

Making use of (2.1) and (2.2) for the wave function then gives (2.3) for the expectation value; with $R\left(r_{1}, r_{2}\right)$ independently normalized, $A$ is independent of the radial portion. The constant $B$ is given by

$$
\begin{aligned}
B= & 2 \sum_{l} \sum_{m} \sum_{m_{1} m_{2}} \sum_{m_{1}^{\prime} m_{2}^{\prime}} \frac{4 \pi}{2 l+1} C\left(l_{1}, l_{2}, L ; m_{1}, m_{2}, M\right) C\left(l_{1}, l_{2}, L ; m_{1}^{\prime}, m_{2}^{\prime}, M\right) \\
& \times\left(\int d \Omega_{1} Y_{l_{2}, m_{2}^{\prime}}^{*}\left(\theta_{1}, \phi_{1}\right) Y_{l_{,}}^{*}\left(\theta_{1}, \phi_{1}\right) Y_{l_{1}, m_{1}}\left(\theta_{1}, \phi_{1}\right)\right) \\
& \times\left(\int d \Omega_{2} Y_{l_{1} m_{1}^{\prime}}^{*}\left(\theta_{2}, \phi_{2}\right) Y_{l, m}\left(\theta_{2}, \phi_{2}\right) Y_{l_{2}, m_{2}}\left(\theta_{2}, \phi_{2}\right)\right)
\end{aligned}
$$

Integrating and eliminating the sum over $m$ with the Kronecker delta that arises then gives

$$
\begin{aligned}
B= & 2 \sum_{l} \sum_{m_{1} m_{2}} \sum_{m_{i}^{\prime}, m_{2}^{\prime}}\left(\frac{2 l_{2}+1}{2 l_{1}+1}\right) C\left(l_{2}, l, l_{1} ; 0,0,0\right)^{2} C\left(l_{1}, l_{2}, L ; m_{1}, m_{2}, M\right) \\
& \times C\left(l_{1}, l_{2}, L ; m_{1}^{\prime}, m_{2}^{\prime} M\right) C\left(l_{2}, l, l_{1} ; M-m_{1}^{\prime}, m_{1}+m_{1}^{\prime}-M, m_{1}\right) \\
& \times C\left(l_{2}, l, l_{1} ; M-m_{1}, m_{1}+m_{1}^{\prime}-M, m_{1}^{\prime}\right) .
\end{aligned}
$$


By making use of the symmetry properties of the Clebsch-Gordan coefficients and the sum rule [13]

$$
\begin{aligned}
C(a, b, e ; \alpha, \beta, \alpha+\beta) C(e, d, c ; \alpha+\beta, \delta, \alpha+\beta+\delta) \\
=\sum_{f}[(2 e+1)(2 f+1)]^{1 / 2} W(a, b, c, d ; e, f) \\
\quad \quad \times C(b, d, f ; \beta, \delta, \beta+\delta) C(a, f, c ; \alpha, \beta+\delta, \alpha+\beta+\delta),
\end{aligned}
$$

and again applying symmetry properties of the coefficients four of the ClebschGordan coefficients can be eliminated through their orthogonality relations [13], with the final result

$$
B=2\left(2 l_{2}+1\right) \sum_{l} C\left(l_{2}, l, l_{1} ; 0,0,0\right)^{2}(-)^{l-L} W\left(l_{2}, l, L, l_{2} ; l_{1}, l_{1}\right) .
$$

The arguments of the Racah coefficient may be interchanged according to

$$
W\left(l_{2}, l, L, l_{2} ; l_{1}, l_{1}\right)=(-)^{l+L-2 l_{1}} W\left(l_{2}, l_{1}, l_{1}, l_{2} ; L, l\right),
$$

which gives

$$
B=2\left(2 l_{2}+1\right) \sum_{l} C\left(l_{2}, l, l_{1} ; 0,0,0\right)^{2} W\left(l_{2}, l_{1}, l_{1}, l_{2} ; L, l\right) .
$$

For the maximum angular momentum of a configuration, $L=l_{1}+l_{2}$, and in this case, $W$ in (7.7) is positive [13] and so $B$ is positive. When $L$ takes on a minimum value of $L=l_{1}-l_{2}$, then $W$ in (7.5) is again positive, and the Clebsch-Gordan coefficient has the form $C\left(l_{2}, l, L+l_{2} ; 0,0,0\right)$. This coefficient vanishes unless the sum of the first three arguments is an even integer, so $l+L$ is even, the coefficient $(-)^{l-L}$ in $(7.5)$ is positive, and $B$ is again positive. Evaluation of the sign of $B$ in the general case has not been accomplished apart from the lowest and highest orbital angular momentum terms of a configuration. We have calculated $B$ for every case occurring in Table II to demonstrate the alternating rule. It is conjectured that the alternating rule is valid in general for the sum (7.7).

\section{Acknowledgments}

This work done in partial fulfillment of the requirements for the Degree of Doctor of Philosophy at the University of Michigan. The authors would like to acknowledge several helpful discussions with Professor T. M. Dunn.

\section{Bibliography}

[1] J. Katriel and R. Pauncz, "Theoretical interpretation of Hund's rule," in Advances in Quantum Chemistry (Academic, New York, 1977), Vol. 10.

[2] J. P. Colpa and M. F. J. Islip, Mol. Phys. 25, 701 (1973).

[3] J. P. Colpa and R. E. Brown, Mol. Phys. 26, 1453 (1973).

[4] A. Lemberger and R. Pauncz, Acta Phys. Acad. Sci. Hungar. 27, 169 (1969).

[5] J. Katriel, Theor. Chim. Acta 26, 163 (1972). 
[6] F. D. Harcourt, J. Beckworth, and R. Geigen, Chem. Phys. Lett. 26, 126 (1974).

[7] R. D. Harcourt and A. Harcourt, Chem. Phys. 1, 238 (1973).

[8] E. R. Davidson, J. Chem. Phys. 42, 4199 (1965).

[9] I. Shim and J. P. Dahl, Theor. Chim. Acta 48, 165 (1978).

[10] J. F. Liebman, Mol. Phys. 21, 563 (1971).

[11] R. T. Brown, J. Chem. Phys. 48, 4698 (1968).

[12] J. C. Slater, Int. J. Quantum. Chem. 4, 561 (1970).

[13] L. C. Biederharn, J. M. Blatt, and M. E. Rose, Rev. Mod. Phys. 24, 249 (1952).

[14] C. E. Moore, Atomic Energy Levels (National Bureau of Standards, Washington, D.C., 1949), Vol. I; Vol. II (1952); Vol. III (1958).

[15] (a) H. N. Russell and W. F. Meggers, Sci. Papers Bur. Stand. 22, 364 (1927); (b) E. U. Condon and G. H. Shortley, The Theory of Atomic Spectra (Cambridge U. P., Cambridge, England, 1953), p. 200.

Received May 29, 1979

Revised October 24, 1979

Accepted for publication February 11, 1980 